\title{
Estudo da dispersão larval radial pós-alimentar em Chrysomya megacephala (Fabricius) (Diptera, Calliphoridae)
}

\author{
Leonardo Gomes ${ }^{1}$ \\ Claudio José Von Zuben ${ }^{1,2}$ \\ Marcos Rogério Sanches ${ }^{3}$
}

\begin{abstract}
Study of postfeeding larval radial dispersal in Chrysomya megacephala (Fabricius) (Diptera, Calliphoridae). Blowflies utilize discrete and ephemeral sites for breeding and larval nutrition. After the exhaustion of food, the larvae begin dispersing to search for sites to pupate or to additional food source, process referred as postfeeding larval dispersal. Some of the most important aspects of this process were investigated in Chrysomya megacephala, utilizing a circular arena to permit the radial dispersion of larvae from the center. To determinate the localization of each pupa, the arena was split in 72 equal sectors from the center. For each pupa, distance from the center of arena, weight and depth were determined. Statistical tests were performed to verify the relation among weight, depth and distance of burying for pupation. It was verified that the larvae that disperse farther are those with higher weights. The majority of individuals reached the depth of burying for pupation between 7 and $18 \mathrm{~cm}$. The study of this process of dispersion can be utilized in the estimation of postmortem interval (PMI) for human corpses in medico-criminal investigations.
\end{abstract}

Keyworos. Blowflies; Calliphoridae; Chrysomya megacephala; forensic entomology; larval dispersal.

\section{INTRODUÇÃO}

As moscas-varejeiras utilizam-se de substratos discretos e efêmeros para posturas dos ovos e para alimentação das larvas (HANSKi 1987; PeSCHKE et al. 1987). O estágio larval é o principal período em que ocorre limitação de recursos alimentares, e a competição por esses recursos é, geralmente, do tipo exploratório (ReIs et al. 1994), em que cada larva procura ingerir o máximo de alimento possível, antes da completa exaustão dos recursos (UlLYETT 1950). Após essa competição, as larvas começam a procurar um sítio para pupação, ou por mais fontes de alimento adicional, no caso daquelas larvas que não obtiveram o peso mínimo para a pupação. O processo é denominado dispersão larval pós-alimentar (GREENBERG 1990).

Chrysomya megacephala (Fabricius), originária da Australásia, foi introduzida acidentalmente no Continente Americano, provavelmente, através de navios (GUIMARÃEs et al. 1978, 1979). Em 1975, esta espécie foi descoberta no Sudeste do Brasil juntamente com C. albiceps (Wiedemann) e C. putoria (Wiedemann) (IMBIRIBA et al. 1977; GuIMARÃes et al. 1978).

As moscas do gênero Chrysomya têm grande importância médico-veterinária por serem veiculadoras de enteropatógenos tais como vírus, bactérias e helmintos (FURLANETTO et al. 1984), podendo causar também miíases nos animais e no homem (Zumpt 1965; Guimarães et al. 1983). São de fundamental importância em entomologia forense por serem indicadoras de tempo de decomposição de cadáveres humanos (Wells \& Greenberg 1992; Von Zuben et al. 1996). O impacto causado com a introdução de espécies exóticas tem interessado aos ecologistas, sendo que a compreensão dos fenômenos biológicos envolvidos neste processo de introdução, tais como competição por recursos alimentares e por sítios de pupação, depende da investigação de determinados parâmetros populacionais, além da estrutura ambiental espaço-temporal em que ocorrem estes fenômenos (Hengeveld 1989). Além desses, ocorrem outros, de pequena escala espacial, como no caso de dispersão dos estágios imaturos dos organismos invasores. No caso do presente estudo, pode ser citada a dispersão larval pós-alimentar de C. megacephala em busca de um sítio para pupação.

O objetivo desse trabalho foi estudar sob condições experimentais, em simulação de ambiente natural, a dispersão

\footnotetext{
1. Departamento de Zoologia, Universidade Estadual Paulista. Av. 24A 1515, 13506-900 Rio Claro-SP, Brasil.

Endereço eletrônico:leugomes@yahoo.com.br

2. Bolsista do CNPq

3. Rua do Rocio 220,10 andar, Vila Olímpia, 04552-903, SP, Brasil. Endereço eletrônico: marcos_sanches@gallup.com
} 


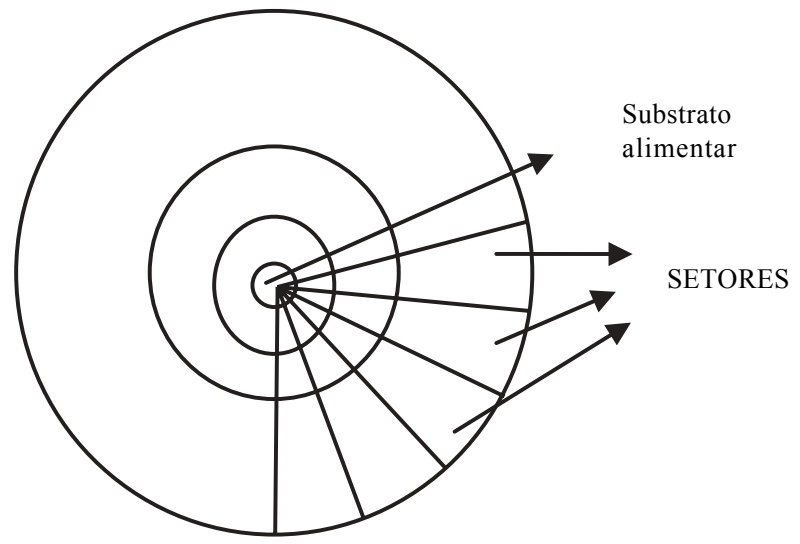

Fig. 1. Esquema geral da arena (protocolo experimental)

larval radial pós-alimentar em C. megacephala e tentar estabelecer uma relação entre as variáveis peso, distância e profundidade de enterramento em uma arena que permitisse às larvas deslocamento em todas as direções. Um dos objetivos, neste caso, foi investigar qual a distância máxima em que as larvas se enterram para empupar.

\section{MATERIALE MÉTODOS}

Exemplares de adultos de C. megacephala foram coletados nos arredores do Instituto de Biociências da UNESP, em Rio Claro, SP, utilizando como isca, matéria orgânica em decomposição (carcaça de peixe). Os exemplares coletados foram identificados até espécie e mantidos em gaiolas teladas,

Tabela I. Valores de profundidade, distância e peso (média e desviopadrão) para 252 pupas de Chrysomya megacephala coletadas em quatro quadrantes da arena (experimento teste).

\begin{tabular}{lcccc}
\hline Quadrante & 1 & 2 & 3 & 4 \\
\hline $\begin{array}{l}\text { Média da } \\
\text { profundidade (cm) }\end{array}$ & 12,19 & 14,88 & 15,37 & 14,36 \\
\hline $\begin{array}{l}\text { Desvio padrão } \\
\text { da profundidade }\end{array}$ & 4,91 & 3,71 & 3,58 & 4,13 \\
\hline $\begin{array}{l}\text { Média da } \\
\text { distância (cm) }\end{array}$ & 13,37 & 12,45 & 13,27 & 15,59 \\
\hline $\begin{array}{l}\text { Desvio padrão } \\
\text { da distância }\end{array}$ & 4,58 & 3,83 & 5,47 & 4,43 \\
\hline $\begin{array}{l}\text { Média do } \\
\text { peso (mg) }\end{array}$ & 4,05 & 3,92 & 4,08 & 3,97 \\
\hline $\begin{array}{l}\text { Desvio padrão } \\
\text { do peso }\end{array}$ & 0,45 & 0,37 & 0,34 & 0,37 \\
\hline
\end{tabular}

em sala com temperatura de $25+1{ }^{\circ} \mathrm{C}, 60 \%$ de umidade relativa e fotofase de 12 horas, sendo ministrados água, açucar e fígado ad libitum.

Uma arena circular foi montada sobre o piso de uma sala do laboratório iluminada de forma homogênea (a fim de evitar tendências na dispersão das larvas), sendo forrada com serragem, tendo suas margens delimitadas por papelão e dividida em 72 setores de 5 graus cada. O desenvolvimento larval da geração $F_{2}$ - por seguir o mesmo tipo de geração de estudos anteriores a fim de evitar diferenças de comportamento no estudo - deu-se em carne moída, sendo este substrato então colocado no centro da arena circular forrada com serragem, para que as larvas abandonassem a mesma e irradiassem para empupar (Fig. 1).

Foram utilizados para esse estudo da dispersão larval radial pós-alimentar, dois experimentos (teste e replicata) com uma arena com $50 \mathrm{~cm}$ de diâmetro e forrada com uma camada de 20 $\mathrm{cm}$ de serragem. A camada de serragem serviu para investigar qual a distância máxima em que as larvas se enterravam para empupar devido ao espaço reduzido para elas se deslocarem na arena como em estudos anteriores em que foi utilizada uma arena de $2 \mathrm{~m}$ de diâmetro (Gomes et al. 2002).

As pupas foram localizadas e retiradas da serragem, sendo determinadas as suas localizações por setor, e medidas, para cada uma, a distância e a profundidade do local de pupação, com o auxílio de régua ou trena. A coleta das pupas começou da periferia da arena para o centro. Cada pupa foi pesada em balança analítica e individualizada em frascos de plástico, antes que ocorresse a emergência do adulto. Os indivíduos adultos foram sexados.

Foram feitos testes estatísticos de regressão e igualdade

Tabela II. Valores de profundidade, distância e peso (média e desviopadrão) para 247 pupas de Chrysomya megacephala em quatro quadrantes da arena de $50 \mathrm{~cm}$ de diâmetro (experimento replicata).

\begin{tabular}{lcccc}
\hline Quadrante & 1 & 2 & 3 & 4 \\
\hline $\begin{array}{l}\text { Média da } \\
\text { profundidade (cm) }\end{array}$ & 12,68 & 13,24 & 12,00 & 14,50 \\
\hline $\begin{array}{l}\text { Desvio padrão } \\
\text { da profundidade }\end{array}$ & 12,88 & 3,16 & 3,69 & 3,18 \\
\hline $\begin{array}{l}\text { Média da } \\
\text { distância (cm) }\end{array}$ & 17,42 & 15,00 & 16,00 & 12,50 \\
\hline $\begin{array}{l}\text { Desvio padrão } \\
\text { da distância }\end{array}$ & 3,50 & 4,64 & 5,17 & 6,85 \\
\hline $\begin{array}{l}\text { Média do } \\
\text { peso (mg) }\end{array}$ & 40,0 & 51,0 & 52,0 & 53,0 \\
\hline $\begin{array}{l}\text { Desvio padrão } \\
\text { do peso }\end{array}$ & 0,70 & 0,65 & 0,50 & 0,44 \\
\hline
\end{tabular}




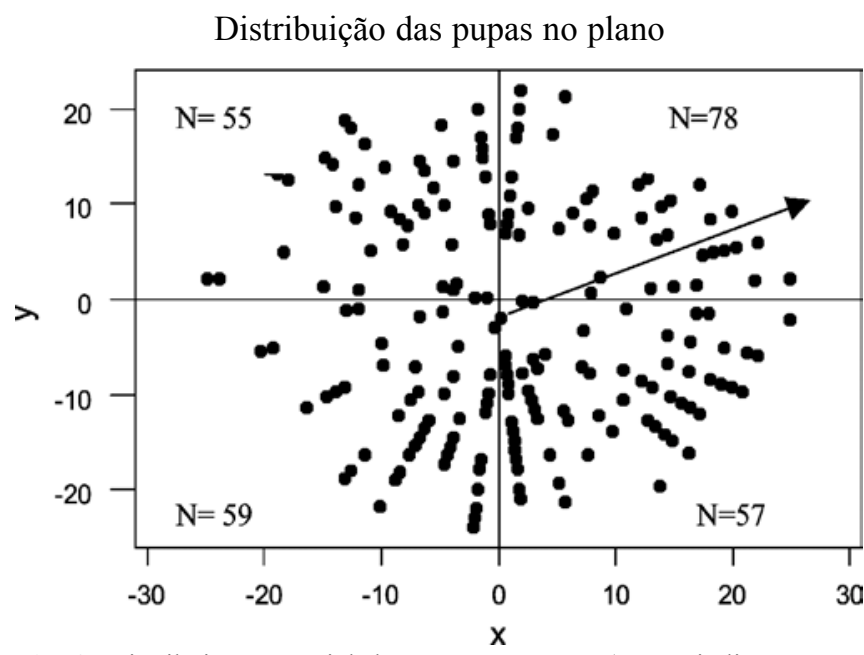

Fig. 2. Distribuição espacial das pupas na arena (a seta indica o vetor direcional médio de distribuição das pupas na arena). $\mathrm{N}=$ número de larvas por quadrante.

de médias a 95\% de confiança e análises direcionais para as variáveis estudadas (ZAR 1999).

\section{RESULTADOS}

Os valores de média e desvio-padrão para profundidade de enterramento, distância em relação ao centro da arena e peso, para as 252 pupas (experimento teste) e 247 (experimento replicata) nos quatro quadrantes da arena estão representados nas Tabelas I e II, respectivamente. Nestas, o quadrante 1 corresponde a setores de 1 a $90^{\circ}$, quadrante 2 , de 91 a $180^{\circ}$, quadrante 3 , de 181 a $270^{\circ}$, e finalmente, quadrante 4 , de 271 a $360^{\circ}$. É preciso ressaltar que, nas ilustrações a seguir, constam os dados dos dois experimentos conjuntamente porque não houve diferença significativa entre os dois grupos (ANOVA, $\mathrm{p}<2,78$ a $5 \%$ de significância).

A Fig. 2 mostra a localização de cada pupa. Pode-se observar que no primeiro quadrante há consideravelmente mais pupas

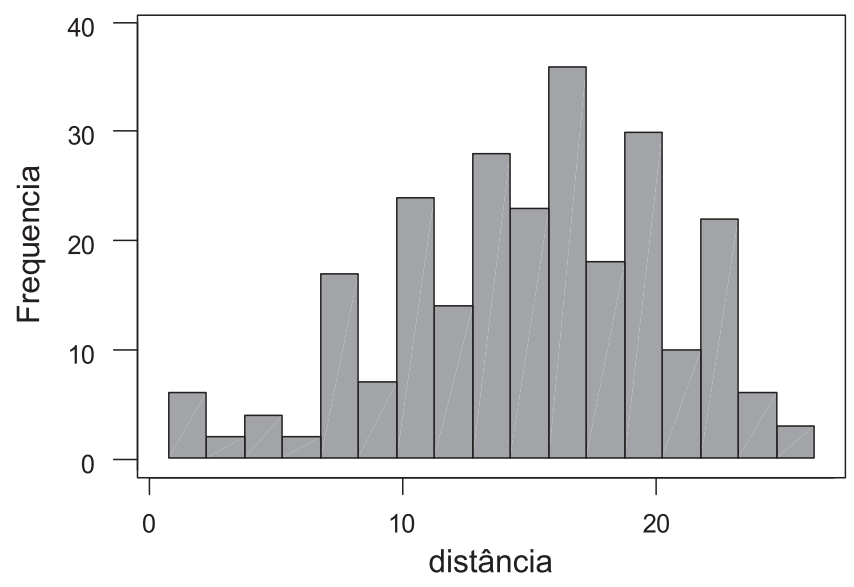

Fig. 3. Distribuição da distância $(\mathrm{cm})$ de enterramento.

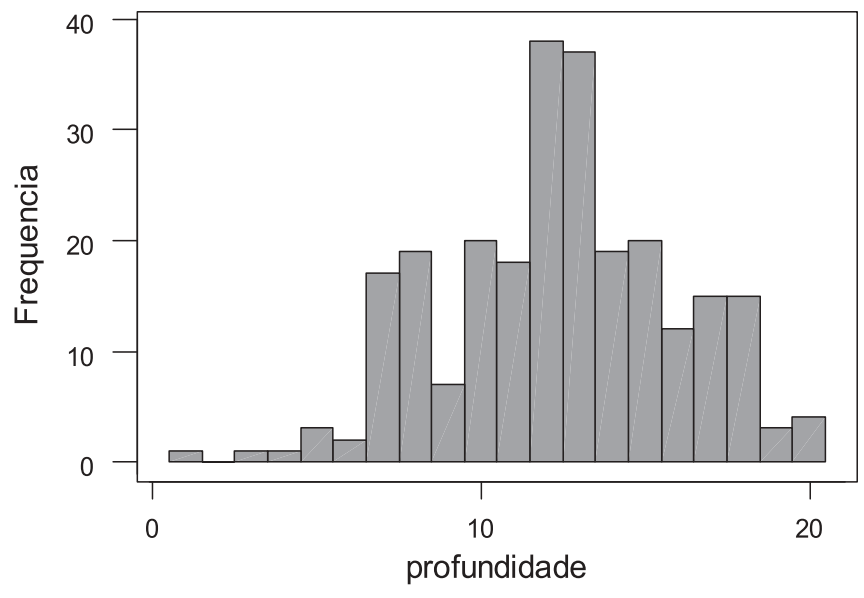

Fig. 4. Distribuição da profundidade $(\mathrm{cm})$ das pupas.

(78 pupas) que nos demais. Se considerarmos o eixo horizontal como 0 grau, as 249 larvas (média dos dois experimentos) tiveram tendência de escolher direções entre $-30^{\circ}$ e $60^{\circ}$. Na Fig. 3, podese observar que houve uma maior concentração de pupas (192 pupas) a partir de $10 \mathrm{~cm}$.

Um resultado interessante é obtido quando se comparam as diversas profundidades em que as larvas se enterraram. De uma forma geral, pode-se observar que não há neste caso muitas larvas enterradas em profundidade máxima (Fig. 4), com quase todas as pupas (180 pupas) concentradas entre 7 e $18 \mathrm{~cm}$.

A distribuição do peso não é tão regular quanto a distribuição da profundidade (Fig. 5). Para o peso há praticamente três modas, que são picos de alta concentração de larvas. Uma ao redor de 40 miligramas, outra ao redor de 50 miligramas e finalmente uma terceira, ao redor de 60 miligramas. Em princípio, pode-se pensar que esta irregularidade na distribuição se deve ao sexo das pupas. As dos machos são, em média, mais leves do que as das fêmeas (machos, 50,737 miligramas e fêmeas, 52,045). De um modo geral, a diferença não é tal que pode-se distinguir o sexo pelo peso das pupas.

Conforme os sexos, tem-se a seguinte distribuição de pupas: $43 \%$ machos, $38 \%$ fêmeas e $18 \%$ indefinido (não emergiu adulto) (Fig. 6). Dentre todas as variáveis, a que mais se relaciona com o sexo das pupas é o coseno do ângulo em que elas se enterraram (Fig. 6).

Na Tabela III são apresentadas algumas correlações entre as variáveis. Pode-se observar que profundidade, distância e peso são correlacionadas positivamente, ou seja, aumentando uma delas, as outras tendem a aumentar também. Pode-se ver também, que o coseno tem correlação negativa com a profundidade e positiva com a distância. Isto indica uma certa evidência de que as larvas que foram para a direita (Fig. 2) tenderam a deslocar-se por maiores distâncias e atingir menores profundidades. A correlação alta entre a coordenada x e o coseno e entre y e seno já era esperada, pois estas variáveis são naturalmente relacionadas, como pode-se ver pela Tabela 3. 


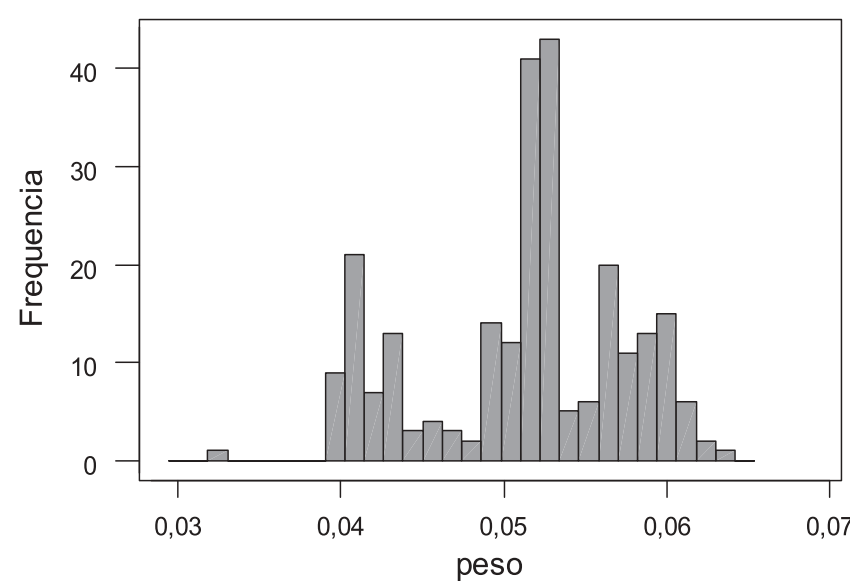

Fig. 5. Distribuição do peso das larvas $\left(\mathrm{em} \mathrm{mg}^{-1}\right)$.

\section{DISCUSSÃO}

O presente estudo demonstrou aspectos gerais sobre o processo de dispersão em uma arena circular, e não confirmou resultados anteriores utilizando arenas com maior diâmetro e menor profundidade, em que as larvas com peso menor são as que têm tendência de se deslocar mais, talvez por estarem à procura de nova fonte alimentar, e não estarem buscando distâncias maiores para se enterrarem para empupar (GoMEs et al. 2002). No presente estudo, observou-se uma correlação positiva entre distância, peso e profundidade das pupas. Isto não confere com o outro experimento (GoMEs et al. 2002) onde observou-se que o peso diminui com a distância. Esta diferença pode ser devida ao fato que, neste experimento, as larvas não puderam se enterrar a grandes distâncias. Se as larvas encontraram a borda e voltaram, isto pode ter feito com que a correlação seja positiva, quando deveria ser negativa como no outro experimento.

A maior concentração de pupas foi observada nas profundidades superiores a $10 \mathrm{~cm}$ e algumas larvas (4 larvas)

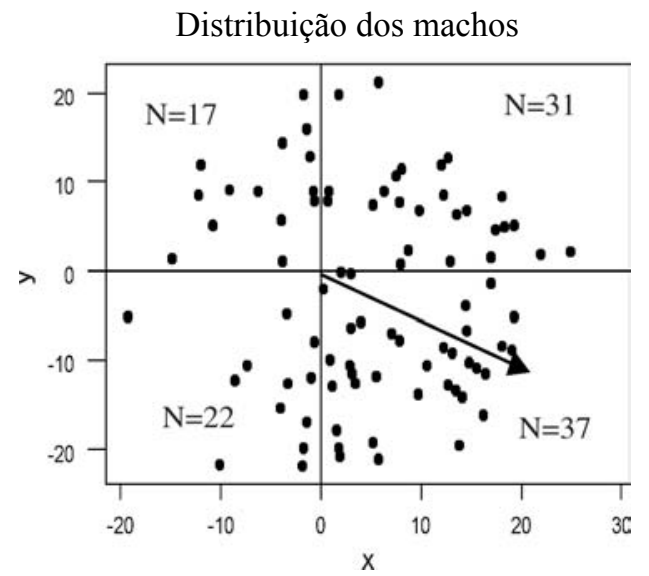

chegaram a atingir a distância máxima possível a partir do centro $(25 \mathrm{~cm})$. Aparentemente, o que mais limitou a movimentação das larvas foi a distância do centro e não a profundidade, embora a utilização de uma arena com essas dimensões $(20 \mathrm{~cm}$ de profundidade e $50 \mathrm{~cm}$ de diâmetro) possibilitou profundidades maiores quando comparado a estudos recentes com uma arena de $2 \mathrm{~m}$ de diâmetro e $5 \mathrm{~cm}$ de profundidade (Gomes et al. 2002). A movimentação das larvas na arena provavelmente ocorreu principalmente em função da busca de sítios para pupação, embora não se possa desconsiderar a possibilidade de alguns indivíduos estarem se movimentando à procura de uma fonte adicional de alimento (Gomes et al. 2002).

Estudos anteriores tinham considerado para o processo de dispersão larval, uma calha de $3 \mathrm{~m}$ de comprimento por 30 $\mathrm{cm}$ de largura, que permitia a locomoção das larvas em apenas uma direção e dois sentidos (Godoy et al. 1995, 1996; BASSANEZI et al. 1997). O presente estudo, ao considerar uma arena circular, reflete melhor as condições de ambiente natural que as larvas enfrentam ao abandonar o substrato alimentar em busca de um sítio para pupação (UlLYeTt 1950). A arena circular permite uma dispersão radial das larvas a partir do substrato alimentar localizado no centro, e não simplesmente uma dispersão numa única direção e apenas dois sentidos, como no caso de uma calha.

Godoy et al. $(1995,1996)$ observaram, tanto para $C$. megacephala (F.) como para C. putoria (Wied.), uma oscilação na freqüência de pupas em função da distância a partir do substrato alimentar. Segundo BoLDRINI et al. (1997), estas oscilações seriam uma conseqüência da formação de agregações larvais em determinado local do substrato de pupação. Acreditase que as larvas dispersantes seriam capazes de perceber a densidade de larvas já enterradas em um determinado ponto do substrato, sendo que aglomerações de larvas num determinado ponto induziriam aquelas que ainda estavam caminhando, a procurar sítios de pupação mais distantes.

O comportamento de dispersão larval pós-alimentar e o conseqüente padrão de distribuição espacial das pupas nos

Fig. 6. Distribuição da posição de enterramento dos machos e das fêmeas (as setas indicam o vetor direcional médio de distribuição das pupas na arena). 
Tabela III. Correlação entre as variáveis.

\begin{tabular}{lllllll}
\hline & $\begin{array}{l}\text { Profun- } \\
\text { didade }\end{array}$ & $\begin{array}{l}\text { Distân- } \\
\text { cia }\end{array}$ & Peso & Cos & Sen & x \\
\hline $\begin{array}{l}\text { Distância } \\
\text { peso }\end{array}$ & 0,185 & & & & & \\
cos & 0,133 & 0,085 & & & & \\
sen & $-0,137$ & 0,135 & $-0,278$ & & & \\
x & 0,082 & $-0,006$ & 0,034 & $-0,079$ & & \\
y & $-0,103$ & 0,095 & $-0,223$ & 0,933 & $-0,062$ & \\
& 0,057 & $-0,074$ & 0,039 & $-0,074$ & 0,949 & $-0,073$ \\
\hline
\end{tabular}

sítios de pupação podem ter implicações na maior ou menor susceptibilidade das mesmas ao ataque de predadores e parasitóides, em populações de ambientes naturais (LEGNER 1977; PeschKe et al. 1987).

É interessante citar que esses estudos de dispersão podem ter importantes implicações para investigações médicocriminais, porque a presença de larvas e pupas nas circunvizinhanças de ou em cadáveres humanos pode auxiliar na estimativa do intervalo de tempo entre a morte e a descoberta do cadáver, referido como intervalo pós-morte (IPM). Essa estimativa constitui-se em aspectos fundamentais em estudos de medicina legal (Sмітн 1986), sendo que a mesma pode ser seriamente prejudicada, levando a uma subestimativa do IPM, caso não sejam consideradas as larvas dispersantes (Von ZuBEN et al. 1998).

\section{CONCLUSÕES}

O presente estudo permitiu concluir que a direção média para os machos é de $-31^{\circ}$ e para as fêmeas, de $243,8^{\circ}$. Como o tamanho do vetor $R$ para as fêmeas é 0,07 e está muito próximo de 0 , não é estatisticamente significante. Assim, quando se considera apenas as pupas fêmeas, não há evidências para dizer que há concentração de pupas em torno da direção média. Por isso, deve-se considerar a hipótese de que a distribuição das direções é uniforme e não há como provar, estatisticamente, que a preferência dos machos é diferente daquela das fêmeas quanto à direção.

A utilização, no presente estudo, de uma arena circular permitindo a locomoção das larvas em todas as direções, permitiu a observação de que pode haver uma maior concentração de larvas em alguns quadrantes desse substrato de pupação. Dessa forma, estudos futuros utilizando técnicas mais detalhadas de análise direcional dos dados, podem vir a confirmar uma tendência de uma maior concentração de larvas deslocando-se em algum sentido preferencial.

Agradecimentos. Os autores do presente trabalho agradecem à FAPESP pelo auxílio financeiro para a realização desses estudos (Processos 98/09939-6 e 00/06336-0).

\section{REFERÊNCIAS}

Bassanezi, R. C.; M. B. F. Leite; W. A. C. Godoy; C. J. Von Zuben; F. J. Von Zuben \& S. F. Reis. 1997. Diffusion model applied to postfeeding larval dispersion in blowflies (Diptera: Calliphoridae). Memórias do Instituto Oswaldo Cruz 92(2): 281-286.

Boldrini J. L.; R. C. Bassanezi; A. C. Moretti; C. J. Von Zuben; W. A. C.Godoy; F. J.Von Zuben \& F. J. Reis. 1997. Nonlocal interactions and the dynamics of dispersal in immature insects. Journal of Theoretical Biology 185: 523-531.

Furlanetto, S. M. P.; M. L. C. Campos; C. M. Hársi; G. M. Buralli \& G. K. Ishinata. 1984. Microrganismos enteropatogênicos em moscas africanas pertencentes ao gênero Chrysomya (Diptera: Calliphoridae) no Brasil. Revista de Microbiologia 15(3): 170-174.

Godoy, W. A. C.; H. G. Fowler; C. J. Von Zuben; L. Ziti \& O. B. Ribeiro. 1995. Larval dispersion in Chrysonya megacephala, C. putoria and Cochliomyia macellaria (Diptera: Calliphoridae). Journal of Applied Entomology 119: 263-266.

Godoy, W. A. C.; S. F. ReIs \& C. J. Von Zuben. 1996. Larval dispersal in Chrysomya megacephala, Chrysomya putoria and Cochliomyia macellaria (Diptera: Calliphoridae): Ecological implications of aggregation behavior. Journal of Applied Entomology 120(4): 423-426.

Gomes, L.; C. J. Von Zuben, J. S. Govone. 2002. Comportamento da dispersão larval radial pós-alimentar em moscas-varejeiras do gênero Chrysomya (Diptera: Calliphoridae): busca por novas fontes de alimento. 2002. Entomologia y Vectores 9(1): 115-132.

Greenberg, B.1990. Behavior of postfeeding larvae of some Calliphoridae and amuscid (Diptera). Annals of the Entomological Society of America 83: 1210-1214.

Guimarães, J. H.; A. P. Prado \& A. X.. Linhares. 1978. Three newly introduced blowfly species in Southern Brazil (Diptera: Calliphoridae). Revista Brasileira de Entomologia 22(1): 53-60.

Guimarães, J. H.; A. P. Prado \& G. M. Buralli 1979. Dispersal and distribution of three newly introduced species of Chrysomya Robineau-Desvoidy in Brazil (Diptera, Calliphoridae). Revista Brasileira de Entomologia 23(4): 245-255.

Guimarães, J. H.; N. Papavero \& A. P. Prado. 1983. As mí́ases na região neotropical (identificação, biologia, bibliografia). Revista Brasileira de Zoologia 1(4): 239-416.

HANSKI, I. 1987. Carrion fly community dynamics: patchiness, seasonality and coexistence. Ecological Entomology 12: 257-266.

Hengeveld, R. 1989. Dynamics of biological invasions. New York, Chapman \& Hall, $160 \mathrm{p}$.

Imbiriba, A. S.; D. T. Izutani; I. T. Milhoreto \& E. Luz. 1977. Introdução da Chrysomya chloropyga (Wiedemann, 1818) na região Neotropical (Diptera: Calliphoridae). Arquivos de Biologia e Tecnologia 20: 35-39.

Legner, E. F. 1977. Temperature, humidity and depth of habitat influencing host destruction and fecundity of muscoid fly parasites. Entomophaga 22: 199-206.

Levot, G. W.; K. R. Brown \& E. Shipp. 1979. Larval growth of some calliphorid and sarcophagid Diptera. Bulletin of Entomological Research 69: 469-475.

Peschke, K.; D. Krapf \& D. Fuldner. 1987. Ecological separation, functional relationships, and limiting resources in a carrion insect community. Zoological Jb. System 114: 241-265,

Reis, S. F.; G. Stangenhaus; W. A. C. Godoy; C. J. Von Zuben \& O. B. Ribeiro. 1994. Variação em caracteres bionômicos em função de densidade larval em Chrysomya megacepala e Chrysomya putoria (Diptera : Calliphoridae). Revista Brasileira de Entomologia 38(1): 3334.

Sмiтн, K. G. V. 1986. A Manual of Forensic Entomology. Ithaca, Cornell University Press.

Ullyett, G. C. Competition for food and allied phenomena in sheepblowfly populations. 1950. Philosophical Transactions of the Royal Society of London B 234: 77-174.

Von Zuben, C. J.; R. C. Bassanezi; S. F. Reis; W. A. C. Godoy \& F. J. V. Zuben. 1996. Theoretical approaches to forensic entomology: I. 
Mathematical model of postfeeding larval dispersal. Journal of Applied Entomology 120(3): 379-382, 1996.

Von Zuben, C. J. 1998. Comportamento de oviposturas individuais, percentagem de eclosão e peso larval mínimo para pupação em populações de Chrysomya megacephala (F.). Anais da Sociedade Entomológica do Brasil 4: 525-533.

Wells, J. D. \& B. Greenberg. 1992. Interaction between Chrysomya rufifacies and Cochliomyia macellaria (Diptera: Calliphoridae): the possible consequences of an invasion. Bulletin of Entomological Research 82: 133-137.

Zar, J. H. 1999. Biostatistical Analysis. Prentice Hall, New Jersey, $666 \mathrm{p}$.

Zumpt, F. 1965. Myiasis in man and animals in the Old World. London, Butterworths, $267 \mathrm{p}$. 